\title{
Virulence and Antibiotic Resistance Profiles of Salmonella Isolated from Chicken Ready Meals and Humans in Egypt
}

\author{
Asmaa G. Mubarak ${ }^{1 *}$, Mona M. Mustafa ${ }^{2}$, Mohamed W. Abdel-Azeem ${ }^{3}$, Dina N. Ali² \\ ${ }^{1}$ Department of Zoonoses, Faculty of Veterinary Medicine, South Valley University, Qena 83523, Egypt; ${ }^{2}$ Assiut \\ Regional Laboratory, Animal Health Institute, Agricultural Research Center; ${ }^{3}$ Department of Microbiology, Faculty \\ of Veterinary Medicine, South Valley University, Qena 83523, Egypt.
}

\begin{abstract}
Salmonellosis is a major public health concern with food economic losses worldwide. This study aimed to investigate the mutual role that may be played by chicken-ready meals and food handlers in the transmission of different Salmonella serotypes to hospitalized patients in Assiut Governorate, Egypt, as well as, to assess their pathogenic potential and antimicrobial resistance. Out of 150 chicken meals collected randomly from various restaurants and food shops including, shish-tawook, pane, and shawerma (50 for each), 10\% were contaminated with Salmonella with the acquisition of shish-tawook (14\%). On the other hand, 100 hand swabs that were assembled from food handlers working in the same places yielded 13 Salmonella isolates, at the time 4 isolates were only obtained from 50 hospitalized patients with diarrhea. From the public health point of view, S. typhimurium, S. enteritidis, and $S$. kentucky were serotyped from both food and human samples. Epidemiologically, insignificant sex risk factor was statistically found in this study although Salmonella was more common in males (14.67\%) than in females (8\%) among food handlers and the opposite among hospitalized patients (4.76\% and $10.34 \%$ in males and females, respectively). Salmonella infection was dominant in $20<35$ and $35<50$ age groups among food handlers and patients, respectively. Complete resistance of the obtained isolates was showed to erythromycin, streptomycin, and nalidixic acid with the highest MAR index (0.640) appeared in clinical isolates from patients compared to food (0.517) and food handlers (0.471). All detected Salmonella serotypes harbored invA gene through which a phylogenetic analysis was conducted for six isolates showing a high degree of similarity between them and those imported from Genbank. hilA, spvC, stn, and qacED1 genes were detected in 75, 16.67, 66.67, and 50\% of Salmonella serotypes, respectively. These findings signify the role played by chicken-ready meals, as well as their handling, in the high rate of multidrug-resistant Salmonella isolates and the risks it poses to public health.
\end{abstract}

Keywords | Chicken meals, Salmonella, Humans, Antibiotics, Virulence, Sequencing

Received | October 26, 2021; Accepted | November 28, 2021; Published | January 10, 2022

*Correspondence | Asmaa G. Mubarak, Department of Zoonoses, Faculty of Veterinary Medicine, South Valley University, Qena 83523, Egypt; Email: a_ mubarek@vet.svu.edu.eg, Asmaa.elgafary@yahoo.com

Citation | Mubarak AG, Mustafa MM, Abdel-Azeem MW, Ali DN (2022). Virulence and antibiotic resistance profiles of Salmonella isolated from chicken-ready meals and humans in Egypt. Adv. Anim. Vet. Sci. 10(2): 377-388.

DOI | http://dx.doi.org/10.17582/journal.aavs/2022/10.2.377.388

ISSN (Online) | 2307-8316

\section{INTRODUCTION}

Salmonellosis remains a major public health issue Worldwide with a huge global burden of morbidity and mortality especially in developing countries (Sodagari et al., 2020). It is estimated to cause 93.8 million human infections and 300,000 deaths annually (WHO, 2020) besides causing a major challenge in the global poultry industry. It is well known that human salmonellosis is associated with the consumption of different kinds of food, in particular poultry and poultry products (Favier et al., 2013). Other routes of infection between individuals are represented by the fecal-oral route and contact with infected pets through contamination of food and drink by the hands, thus disease outbreaks can occur (Munck et al., 2020). 
Chicken meat and its products are characterized by deliciousness, nutritiousness, good flavor, and easily digested which make them very popular foods throughout the world, therefore poultry is a predominant source of foodborne illnesses (Chai et al., 2017). Over 2600 different Salmonella serotypes have been identified, 2000 of them can be found in chickens (Takaya et al., 2020). So, chickens have been implicated in most Salmonella outbreaks.

Salmonella recovered from chickens can be differentiated into three groups. The first group includes highly hostadapted and invasive serotypes such as $S$. typhi in humans, S. gallinarum and S. pullorum in poultry. The second is non-host adapted and invasive serotypes as $S$. typhimurium, $S$. arizonae, and $S$. enteritidis. The third group contains non-host adapted and non-invasive serotypes, which are mostly harmless to animals and humans (Andino and Hanning, 2015).

Salmonella pathogenicity has been related to many virulence genes existent in the chromosomal Salmonella pathogenicity islands (SPIs) (Nayak et al., 2004). The invA gene is essential for epithelial cells invasion (E1-Sharkawy et al., 2017) and has been established to be present in Salmonella species only, hence it is used in the genetic diagnosis of Salmonella species (Fekry et al., 2018). From medical and pharmaceutical points of view inv $A$ gene can help in developing specific medicines against salmonella (Almas et al., 2021). Whereas, an operon spo harbors five genes sprRABCD and is commonly associated with some serotypes initiating the systemic spread of the pathogen. The spuC is a virulence-related gene on the plasmid required for survival within the host cell (Card et al., 2016). HilA gene is required to induce apoptosis of macrophages and invade epithelial cells (Borges et al., 2013). Besides, Salmonella enterotoxin (stn) gene which codes for enterotoxin production and is a causative agent of diarrhea (Xu et al., 2010).

Salmonellosis is often characterized clinically by nausea, vomiting, abdominal cramps, and diarrhea which is usually a self-limiting disease, but complications and deaths have been recorded especially in children, the elderly, and immunocompromised persons (Nayak et al., 2004). The increasing trend of Salmonella multi-drug resistance is mainly associated with the overuse of antibiotics in treating Salmonella infections and incorporation of growth promoters in animal feed (Ed-dra et al., 2017) causing a public health threat. In recent days, Salmonella strains showed increased resistance to several antibiotics comprising of $\beta$-lactams, cephalosporins, and non- $\beta$-lactam antimicrobials as tetracyclines, quinolones, sulfonamides, and polymyxins (das Neves et al., 2020; Rodrigues et al., 2020).
Meanwhile, resistance to the quaternary ammonium compounds (QACs) has been developed, which are cationic surface-active detergents, representing disinfectants of choice widely used in the poultry industry due to their low relative toxicity, good antibacterial properties, and noncorrosive to reduce or eliminate potentially pathogenic microbial loads (Haynes and Smith, 2003). The resistance to those disinfectants might be caused by intrinsic factors, with increased tolerance of the bacteria due to repeated exposure, or developed through genetic change. Likewise, there is evidence of the occurrence of cross-resistance and co-resistance between widely used disinfectants and antibiotics (Techaruvichit et al., 2016; Bakheet et al., 2017). The disinfectant resistance genes are commonly located in mobile genetic elements, four genes of QAC ( $q a \mathrm{E}$, $q a c F$, qac $G$ and $\operatorname{sug} E(p)$ ) have been identified (Zou et al., 2014).

Therefore, this study aimed to assess the incidence, serotyping, virulence genes, and associations of Salmonella resistance recovered from chicken-ready meals, food handlers, and hospitalized patients in Assiut Governorate, Egypt.

\section{MATERIALS AND METHODS}

\section{STUDY DESIGN AND SAMPLING}

This study was conducted in Assiut Province during the period between 2018 and 2020 during which a total number of 150 chicken-ready meals were collected randomly from restaurants, food shops, and street vendors including shish-tawook, pane, and shawerma (50 for each). The samples were purchased in sterile and sealed plastic containers and transferred immediately to the laboratory for further processing. Twenty-five grams of each sample was added to $225 \mathrm{~mL}$ of buffered peptone water (BPW) (Himedia, India) and mixed well by using a homogenizer, then incubated at $37{ }^{\circ} \mathrm{C}$ for $18-24$ hours (Gracias and McKillip, 2004).

On the other hand, 150 human samples were collected, represented by 100 hand swabs which were collected from food handlers in the same restaurants and food shops, by dipping sterile cotton swabs into saline-containing sterile test tubes and then rubbing under fingernails, the palm of the hands, and between fingers. As well as 50 diarrhea samples from patients who suffered from gastrointestinal disturbances with diarrhea, who admitted to Abo-Noub Hospital, Assiut, Egypt were collected in clean cups. Then all samples were transferred to tubes contained BPW (Himedia, India) and incubated at $37^{\circ} \mathrm{C}$ for $18-24$ hours. 
ISOLATION AND IDENTIFICATION OF SALMONELLA (ISO, 2002)

Pre-inoculated BPW were transferred to $10 \mathrm{ml}$ RappaportVassiliadis Soya (RVS) broth (Himedia, India) as selective enrichment and incubated at $42^{\circ} \mathrm{C}$ for 24 hours. Then a loopful was streaked on Xylose Lysine Deoxycholate (XLD) agar (Himedia, India) and incubated overnight at $37^{\circ} \mathrm{C}$. Typical colonies were picked and biochemically tested by standard devices as urease, sugar fermentation, methyl-red, Voges-Proskauer, indole, and citrate tests.

\section{SERotyping OF SALMoNELLA ISOLATES}

Biochemically identified Salmonella isolates were serotyped to determine somatic $(\mathrm{O})$ and flagellar $(\mathrm{H})$ antigens using Salmonella antisera (Denka Seiken Co., Japan) according to Kauffman White scheme (Kauffman, 1974).

\section{Phenotypic Detection of Antibiotic Resistance}

The antibiotic resistance test was performed using the disc diffusion method on Muller-Hinton according to the National Committee for Clinical Laboratory Standards (CLSI, 2017). The following antibiotics were assessed $(\mu \mathrm{g} / \mathrm{ml})$ : Oxytetracycline (T, 30), Ciprofloxacin (CP, 5), Cephalothin (CN, 30), Neomycin (N, 30), Erythromycin (E, 15), Nalidixic acid (NA, 30), Ampicillin (AM, 10), Cephradine(CE,30),Doxycycline(DO,30),Kanamycin(K, 30), Streptomycin (S, 10), Cefotaxim (CF, 30), Gentamicin $(\mathrm{G}, 10)$, Amikacin (AK, 30), Sulphamethoxazol (SXT, 25), and Penicillin G (P, 10 IU).

\section{DETECTION OF SOME VIRULENCE GENES}

Salmonella serotypes obtained in this study were screened for the presence of some virulence and qacED1 disinfectant genes using PCR. The primers used were presented in Table 1.

\section{DNA EXTRACTION}

Genomic DNA was extracted from Salmonella cultures using GeneJET Genomic DNA Purification Kit (Fermentas) following the manufacturer's instructions. The extracted DNA was stored at $-20^{\circ} \mathrm{C}$ till further use.

\section{Amplification of Virulence genes by Multiplex PCR}

PCR amplification was performed using a thermal cycler (Master cycler, Eppendorf, Hamburg, Germany) following the manufacturer's instruction. The thermocycling conditions consisted of initial denaturation cycle at $94^{\circ} \mathrm{C}$ for $2 \mathrm{~min}, 30$ cycles of denaturation at $94^{\circ} \mathrm{C}$ for $45 \mathrm{sec}$, annealing at $53^{\circ} \mathrm{C}$ for $1 \mathrm{~min}$, extension at $72^{\circ} \mathrm{C}$ for $1 \mathrm{~min}$ and final extension at $72^{\circ} \mathrm{C}$ for $7 \mathrm{~min}$. Amplified DNA fragments were resolved by gel electrophoresis using 1.5 $\%(\mathrm{w} / \mathrm{v})$ agarose stained with ethidium bromide solution
$(0.5 \mu \mathrm{g} / \mathrm{ml})$, visualized under an ultraviolet transilluminator and photographed.

\section{AMPLIFICATION OF QACED1 GENE OF SALMONELLA}

The PCR cycling protocol was applied as following, An initial denaturation at $94^{\circ} \mathrm{C}$ for $60 \mathrm{sec}$, followed by 35 cycles of denaturation at $94^{\circ} \mathrm{C}$ for $60 \mathrm{sec}$, annealing at $64^{\circ} \mathrm{C}$ for $30 \mathrm{sec}$ and extension at $72^{\circ} \mathrm{C}$ for $30 \mathrm{sec}$, followed by a final extension at $72^{\circ} \mathrm{C}$ for $7 \mathrm{~min}$. Finally, $5 \mu \mathrm{l}$ of each amplicon was electrophoresed in 1\% agarose gel stained with ethidium bromide, visualized and captured on UV transilluminator.

\section{Sequencing INVA Gene}

Purified PCR products using QIA quick extraction kit (Qiagen, Valencia, CA) of inv $A$ gene from six isolates; three of food origin (S. kentucky_CH1, S. typhimurium_CH2, S. enteritidis_CH3); two from hand swabs (S. kentucky $\mathrm{H} 1$ and $S$. typhimurium_H2) and one from diarrhea ( $S$. enteritidis_H3) were sequenced on an Applied Biosystems 3130 Sequencer (ABI, USA) using Bigdye Terminator V3.1 cycle sequencing kit (Perkin-Elmer). A BLAST ${ }^{\circledR}$ analysis (Basic Local Alignment Search Tool) (Altschul et al., 1990) was initially performed to establish sequence identity to GenBank. Purification of the sequence reaction occurred by using Centrisep (spin column), Cat. No. CS901 of 100 reactions according to the manufacturer's instruction.

\section{Phylogenetic analysis}

The obtained sequences were subjected to BLAST similarity and phylogenetic analysis using the neighbor joining method on Mega 6 program (Tamura et al., 2013).

\section{STATISTICAL ANALYSis}

Data were statistically analyzed using a SPSS version 22, Pearson chi-square test, Fisher's exact test, and Monte Carlo test were used to predicate the association between variables followed by Contingency coefficient/ Phi correlation. Finally, Eta square ( $\eta 2$ ) was applied to measure the effect size of variance. To establish the risk factors, Mantel-Haenszel statistics were computed once for all variables and odd ratio between two dichotomous factor variables to measure the strength of the association.

\section{RESULTS AND DISCUSSION}

INCIDENCE OF SALMONELLA IN CHICKEN-READY MEALS In this study, a series of devices were conducted for isolation, identification of Salmonella species and detection of their virulence and resistance. Out of the examined 150 chickenready meals, 15 (10\%) were contaminated with Salmonella with the higher incidence in shish-tawook 14\% (7/50) 
followed by $10 \%(5 / 50)$ in panee, and the lowest incidence was detected in shawerma samples 6\% (3/50) without significant difference $(P=0.411)$, while a weak correlation between the chicken meals and Salmonella contamination $(c=0.108)$ was found with small effect size $(\eta 2=0.012)$ as clarified in Table 2. Serotyping of the isolates revealed that S. kentucky and S. typhimurium were typed in the same percentage of 20 (3/15), also S. enteritidis and $S$. molade were equally serotyped as $13 \%(2 / 15)$, while $S$. inganda, $S$. tamale, S. larochelle, $S$. tsevie, and $S$. wingrove were typed as $7 \%(1 / 15)$ for each with statistically significant difference $(\wedge=0.000)$ (Table 3$)$.
INCIDENCE OF SALMONELLA IN HUMAN SAMPLES

Data illustrated in Table 2 clarified that out of 100 and 50 examined hand swabs and diarrhea samples, 13 and 4 Salmonella isolates were obtained, respectively with insignificant association $(P=0.362)$, and weak correlation $\left(0.074^{\mathrm{P}}\right)$. Seventeen human Salmonella isolates obtained in this study revealed six different serovars which significantly differ $(P=0.000 \mathrm{~b})$ with $S$. enteritidis was the predominant 6 (35.3\%), while S. ttyphimurium and $S$. infantis were the most frequent as $4(24 \%)$ and $3(18 \%)$, respectively. Whereas S. kentucky, S. risen and S. heidelberg represented as $1(6 \%)$ for each, one untypable isolate was found (6\%) from hand swabs as outlined in Table 4.

Table 1: Primer sequences of Salmonella genes.

\begin{tabular}{|c|c|c|c|}
\hline Target gene & Oligonucleotide sequence $\left(5^{\prime} \rightarrow 3^{\prime}\right)$ & Product size (bp) & Reference \\
\hline invA & $\begin{array}{l}\text { TATCGCCACGTTCGGCAA } \\
\text { TCGCACCGTCAAAGGAACC }\end{array}$ & 275 & Nayak et al. (2004) \\
\hline hilA & $\begin{array}{l}\text { CGGAAGCTTATTTGCGCCATGCTGAGGTAG } \\
\text { GCATGGATCCCCGCCGGCGAGATTGTG }\end{array}$ & 854 & Castro et al. (2002) \\
\hline spvC & $\begin{array}{l}\text { CGGAAATACCATCAAATA } \\
\text { CCCAAACCCATACTTACTCTG }\end{array}$ & 669 & Swamy et al. (1996) \\
\hline $\operatorname{stn}$ & $\begin{array}{l}\text { TTGTGTCGCTATCACTGGCAACC } \\
\text { ATTCGTAACCCGCTCTCGTCC }\end{array}$ & 617 & Murugkar et al. (2003) \\
\hline qacED1 & $\begin{array}{l}\text { TAAGCCCTACACAAATTGGGAGATAT } \\
\text { GCC TCC GCA GCG ACT TCCACG }\end{array}$ & 62 & Chuanchuen et al. (2007) \\
\hline
\end{tabular}

Table 2: Incidence of Salmonella in the examined samples.

\begin{tabular}{|c|c|c|c|c|c|c|}
\hline $\begin{array}{l}\text { Sources of } \\
\text { samples }\end{array}$ & $\begin{array}{l}\text { No. of exam- } \\
\text { ined samples }\end{array}$ & $\begin{array}{l}\text { +ve Salmonella } \\
\text { No. }(\%)\end{array}$ & $\begin{array}{l}\text { Pearson Chi- } \\
\text { Square } X^{2}(P)\end{array}$ & $\begin{array}{l}\text { Contingency/Phi } \\
\text { coefficient } R(P)\end{array}$ & $\begin{array}{l}\text { Eta squared } \\
\text { value }(\eta 2)\end{array}$ & Odd ratio value \\
\hline \multicolumn{7}{|c|}{ Chicken-ready meals } \\
\hline Shish tawook & 50 & $7(14)$ & \multirow[t]{5}{*}{$1.778(0.411)$} & \multirow[t]{5}{*}{$0.108^{\mathrm{C}}(0.411)$} & \multirow[t]{5}{*}{0.012} & $0.392(0.095-1.613)$ \\
\hline Panee & 50 & $5(10)$ & & & & $0.574(0.130-2.545)$ \\
\hline Shawerma & 50 & $3(6)$ & & & & Reference \\
\hline Total & 150 & $15(10)$ & & & & \\
\hline \multicolumn{4}{|l|}{ Human samples } & & & \\
\hline Hand swab & 100 & $13(13)$ & \multirow[t]{4}{*}{$0.829(0.362)$} & \multirow[t]{4}{*}{$0.074^{\mathrm{P}}(0.362)$} & \multirow[t]{3}{*}{0.006} & \multirow[t]{3}{*}{$1.718(0.530-5.571)$} \\
\hline Diarrheal swab & 50 & $4(8)$ & & & & \\
\hline Total & 150 & $17(11.33)$ & & & & \\
\hline Over all total & 300 & $32(10.67)$ & & & & \\
\hline
\end{tabular}

${ }^{\mathrm{C}}$ : contingency coefficient; ${ }^{\mathrm{P}}$ : Phi coefficient.

Table 3: Serotyping of Salmonella isolates from chicken-ready meals.

\begin{tabular}{|c|c|c|c|c|c|c|c|c|c|c|c|c|}
\hline \multirow[t]{2}{*}{$\begin{array}{l}\text { Sources of } \\
\text { samples }\end{array}$} & \multirow[t]{2}{*}{$\begin{array}{l}\text { No. of } \\
\text { isolates }\end{array}$} & $\begin{array}{l}\text { S.ken- } \\
\text { tucky }\end{array}$ & $\begin{array}{l}\text { S. typhi- } \\
\text { murium }\end{array}$ & $\begin{array}{l}\text { S. enter- } \\
\text { itidis }\end{array}$ & $\begin{array}{l}\text { S. } \\
\text { molade }\end{array}$ & $\begin{array}{l}\text { S. } \\
\text { inganda }\end{array}$ & $\begin{array}{l}S . \\
\text { tamale }\end{array}$ & $\begin{array}{l}\text { S. } \\
\text { larochelle }\end{array}$ & S. tservie & $\begin{array}{l}\text { S. win- } \\
\text { grove }\end{array}$ & $\begin{array}{l}\text { Fishers } \\
\text { exact test }\end{array}$ & $\begin{array}{l}\text { Eta } \\
\text { squared }\end{array}$ \\
\hline & & No. (\%) & No. (\%) & No. (\%) & No. (\%) & No. (\%) & No. (\%) & No. (\%) & No.(\%) & No. (\%) & & \\
\hline Shish-tawook & 7 & $1(14)$ & $2(29)$ & $1(2)$ & $1(14)$ & $1(14)$ & $1(14)$ & $0(0)$ & $0(0)$ & $0(0)$ & $0.000^{\wedge}$ & \\
\hline Panee & 5 & $1(20)$ & $1(20)$ & $0(0)$ & $1(20)$ & $0(0)$ & $0(0)$ & $1(20)$ & $1(20)$ & $0(0)$ & $0.000^{\wedge}$ & \\
\hline Shawarma & 3 & $1(33)$ & $0(0)$ & $1(2)$ & $0(0)$ & $0(0)$ & $0(0)$ & $0(0)$ & $0(0)$ & $1(33)$ & $0.000^{\wedge}$ & 0.841 \\
\hline Total & 15 & $3(20)$ & $3(20)$ & $2(13)$ & $2(13)$ & $1(7)$ & $1(7)$ & $1(7)$ & $1(7)$ & $1(7)$ & & \\
\hline
\end{tabular}

$\wedge$ : fisher's exact test. 
Table 4: Serotyping of Salmonella isolates from human samples.

\begin{tabular}{|c|c|c|c|c|c|c|c|c|c|c|}
\hline $\begin{array}{l}\text { Sources of } \\
\text { sample }\end{array}$ & $\begin{array}{l}\text { No. of } \\
\text { isolates }\end{array}$ & $\begin{array}{l}\text { S. enter- } \\
\text { itidis }\end{array}$ & $\begin{array}{l}\text { S. typhi- } \\
\text { murium }\end{array}$ & $\begin{array}{l}\text { S. infan- } \\
\text { tis }\end{array}$ & $\begin{array}{l}\text { S. ken- } \\
\text { tucky }\end{array}$ & S. rissen & $\begin{array}{l}\text { S. heidel- } \\
\text { berg }\end{array}$ & Untypable & Monte carlo test & $\begin{array}{l}\text { Eta } \\
\text { squared }\end{array}$ \\
\hline & & No. (\%) & No. (\%) & No. $(\%)$ & No. $(\%)$ & No. (\%) & No. $(\%)$ & No. (\%) & Value $(P)$ & \\
\hline Hand swabs & 13 & $5(39)$ & $2(15)$ & $2(15)$ & $1(8)$ & $1(8)$ & $1(8)$ & $1(8)$ & $72.535(0.000 \mathrm{~b})$ & \multirow[t]{3}{*}{0.885} \\
\hline Diarrheal swabs & 4 & $1(25)$ & $2(50)$ & $1(25)$ & $0(0)$ & $0(0)$ & $0(0)$ & $0(0)$ & $26.398(0.000 \mathrm{~b})$ & \\
\hline Total & 17 & $6(35.3)$ & $4(24)$ & $3(18)$ & $1(6)$ & $1(6)$ & $1(6)$ & $1(6)$ & & \\
\hline
\end{tabular}

b: Monte Carlo test.

Table 5: Incidence of Salmonella in humans stratified by sex and age.

\begin{tabular}{|c|c|c|c|c|c|c|c|c|}
\hline \multirow[t]{2}{*}{ Variable } & \multicolumn{2}{|c|}{ Hand swabs (No.=100) } & \multicolumn{2}{|c|}{ Diarrheal swabs $($ No. $=50)$} & \multirow{2}{*}{$\begin{array}{l}\text { Fishers } \\
\text { exact test } \\
\text { P value }\end{array}$} & \multirow{2}{*}{$\begin{array}{l}\text { Mantel-haenszel } \\
\text { odds ratio } \\
\text { Value }\end{array}$} & \multirow{2}{*}{$\begin{array}{l}\text { Eta } \\
\text { squared } \\
\text { Value }\end{array}$} & \multirow{2}{*}{$\begin{array}{l}\text { Odds ratio } \\
\text { Value }\end{array}$} \\
\hline & $\begin{array}{l}\text { No. of exam- } \\
\text { ined samples }\end{array}$ & $\begin{array}{l}\text { +ve Salmonella } \\
\text { No. }(\%)\end{array}$ & $\begin{array}{l}\text { No. of exam- } \\
\text { ined samples }\end{array}$ & $\begin{array}{l}\text { +ve Salmonella } \\
\text { No. }(\%)\end{array}$ & & & & \\
\hline \multicolumn{9}{|c|}{ Patient sex } \\
\hline Male & 75 & $11(14.67 \%)$ & 21 & $1(4.76)$ & 0.454 & \multirow{3}{*}{$\begin{array}{l}1.674(0.464- \\
6.035)\end{array}$} & \multirow{3}{*}{0.002} & \multirow{3}{*}{$1.4(0.465-4.211)$} \\
\hline Female & 25 & $2(8)$ & 29 & $3(10.34)$ & 1.000 & & & \\
\hline \multicolumn{6}{|c|}{ Patient age (years) } & & & \\
\hline $5<20$ & 7 & $0(0)$ & 16 & $1(6.25)$ & 1.000 & \multirow{4}{*}{$\begin{array}{l}1.388(0.399- \\
4.832)\end{array}$} & \multirow[t]{4}{*}{0.000} & Reference \\
\hline $20<35$ & 63 & $10(15.87)$ & 20 & $2(10)$ & 0.721 & & & $\begin{array}{l}2.2(0.0185- \\
26.157)\end{array}$ \\
\hline $35<50$ & 15 & $1(6.67)$ & 7 & $1(14.29)$ & 1.000 & & & $\begin{array}{l}0.592(0.122- \\
2.864)\end{array}$ \\
\hline $50<60$ & 15 & $2(13.33)$ & 7 & $0(0)$ & 1.000 & & & $1(0.128-7.812)$ \\
\hline
\end{tabular}

Data in Table 5 elucidated that Salmonella incidence in hand swabs differed with $14.67 \%$ among males in contrast to $8 \%$ among females, contrarily to its incidence in diarrhea samples which were $4.76 \%$ and $10.34 \%$ in males and females, respectively without significant values. Insignificantly, hand swabs collected from the age group of $20<35$ showed the highest incidence of Salmonellosis $(15.87 \%)$ followed by ages of $50<60(13.33 \%)$, finally the age group of $35<50(6.67)$, while couldn't be detected in $5<20$ age group. Patients from whom the highest positive diarrheal samples were collected ranged from $35<50$ years old $(14.29 \%)$ followed by $20<35$ (10\%) then $5<20$ (6.25\%). None of the diarrhea samples collected from the oldest age $(50<60)$ showed Salmonella infection.

\section{ANTIBIOTIC RESISTANCE PROFILE OF FOOD ISOLATES}

The results of Salmonella isolates resistance to sixteen antibiotics were given in Table 6. Salmonella isolates originated from chicken meals were all resistant to erythromycin and streptomycin (100\%) followed by cephradine (93.3\%), low frequency of resistance was observed to ampicillin (6.7\%) and doxycycline (13.3\%). As can be seen in Table 7 , a high MAR index $(0.125-1)$ was observed in Salmonella isolated from ready meals with the highest index value of 1 was found in one $S$. Kentucky isolate. Most Salmonella isolates were multi-drug resistant (MDR) to at least three antibiotics (erythromycin, streptomycin, and cephradine) with one $S$. tamale isolate was resistant to erythromycin and streptomycin only.

Table 6: Frequency of antibiotic resistance of Salmonella isolates.

\begin{tabular}{|llll} 
Antibiotics & $\begin{array}{l}\text { Food } \\
\text { strains } \\
\text { (No.=15) }\end{array}$ & $\begin{array}{l}\text { Hand swabs } \\
\text { strains } \\
\text { (No.=13) }\end{array}$ & $\begin{array}{l}\text { Diarrheal } \\
\text { strains } \\
\text { (No.=4) }\end{array}$ \\
& No. (\%) & No. (\%) & No. (\%) \\
Erythromycin (E) & $15(100)$ & $12(92.3)$ & $4(100)$ \\
\hline Streptomycin (S) & $15(100)$ & $13(100)$ & $4(100)$ \\
Cephradine (CE) & $14(93.3)$ & $12(92.3)$ & $3(75.0)$ \\
Sulphamethoxazol (SXT) & $12(80)$ & $7(53.8)$ & $3(75.0)$ \\
Cephalothin (CN) & $11(73.3)$ & $6(46.1)$ & $3(75.0)$ \\
Nalidixic acid (NA) & $9(60)$ & $9(69.2)$ & $4(100)$ \\
Cefotaxim (CF) & $8(53.3)$ & $5(38.5)$ & $3(75.0)$ \\
Penicillin G (P) & $6(40)$ & $8(61.5)$ & $3(75.0)$ \\
\hline Neomycin (N) & $6(40)$ & $4(30.8)$ & $3(75.0)$ \\
\hline Oxytetracycline (T) & $6(40)$ & $6(46.1)$ & $2(50.0)$ \\
Kanamycin (K) & $6(40)$ & $4(30.8)$ & $2(50.0)$ \\
Gentamicin (G) & $5(33.3)$ & $1(7.7)$ & $1(25.0)$ \\
\hline Amikacin (AK) & $5(33.3)$ & $2(15.4)$ & $2(50.0)$ \\
\hline Ciprofloxacin (CP) & $3(20)$ & $3(23.1)$ & $1(25.0)$ \\
\hline Doxycycline (DO) & $2(13.3)$ & $4(30.8)$ & $2(50.0)$ \\
\hline Ampicillin (AM) & $1(6.7)$ & $2(15.4)$ & $1(25.0)$ \\
\hline
\end{tabular}




\section{OPEN OACCESS}

ANTIBIOTIC RESISTANCE PROFILE OF HUMAN ISOLATES Table 6 showed that all Salmonella isolates obtained from food handlers pronounced a complete resistance to streptomycin with higher resistance to erythromycin and cephradine (92.3\%). Contrarily, gentamicin was the most effective against Salmonella followed by amikacin and ampicillin with resistance rates of 7.7, 15.4, and 15.4\%, respectively. One $S$. enteritidis isolate exhibited MAR index of 1, all isolates were MDR to three antibiotics (erythromycin, streptomycin, and cephradine) or more as clarified in Table 8.
About diarrheal isolates, Table 6 displayed a complete resistance to erythromycin, streptomycin, and nalidixic acid followed by cephradine, sulphamethoxazol, cephalothin, cefotaxim, penicillin $\mathrm{G}$, and neomycin with the same resistance rate of $75.0 \%$, low level of resistance was detected to gentamicin, ciprofloxacin, and ampicillin (25.0\%). Constantly, data recorded in Table 9 exposed that one S. typhimurium isolate showed MAR index of 1 with MDR in all isolates exhibiting resistance to three antibiotics (erythromycin, streptomycin, and nalidixic acid) or more.

Table 7: Antibiotic resistance profile of food Salmonella isolates.

\begin{tabular}{|c|c|c|c|}
\hline No. & Salmonella serotype & Antimicrobial resistance profile & MAR index \\
\hline 1 & S. kentucky & E, S, CE, SXT, CN, NA, CF, P, N, T, K, G, AK, CP, DO, AM & 1 \\
\hline 2 & S. kentucky & E, S, CE, SXT, CN, NA, CF, P, N, T, K & 0.688 \\
\hline 3 & S. kentucky & $\mathrm{E}, \mathrm{S}, \mathrm{CE}$ & 0.188 \\
\hline 4 & S. enteritidis & E, S, CE, SXT, CN, NA, CF, P, N, T, K, G, AK, CP, DO & 0.938 \\
\hline 7 & S. enteritidis & E, S, CE, SXT & 0.250 \\
\hline 8 & S. typhimurium & E, S, CE, SXT, CN, NA, CF, P, N, T, K, G, AK, CP & 0.875 \\
\hline 9 & S. typhimurium & E, S, CE, SXT, CN, NA, CF & 0.438 \\
\hline 9 & S. typhimurium & E, S, CE, SXT, CN, NA, CF & 0.438 \\
\hline 10 & S. molade & E, S, CE, SXT, CN, NA, CF, P, N, T, K, G, AK & 0.813 \\
\hline 11 & S. molade & E, S, CE, SXT, CN & 0.313 \\
\hline 12 & S. wingrove & E, S, CE, SXT, CN, NA, CF, P, N, T, K, G, AK & 0.813 \\
\hline 13 & S. larochelle & $\mathrm{E}, \mathrm{S}, \mathrm{CE}, \mathrm{SXT}, \mathrm{CN}, \mathrm{NA}$ & 0.375 \\
\hline 14 & S. tsevie & E, S, CE, SXT, CN & 0.313 \\
\hline 15 & S. inganda & $\mathrm{E}, \mathrm{S}, \mathrm{CE}$ & 0.188 \\
\hline 16 & S. tamale & $\mathrm{E}, \mathrm{S}$ & 0.125 \\
\hline
\end{tabular}

MAR index $=$ No. of resistance $/$ Total No. of tested antibiotics.

Table 8: Antibiotic resistance profile of hand swabs Salmonella isolates.

$\begin{array}{llll}\text { NO } & \text { Salmonella } \text { strains } & \text { Antimicrobial resistance profile } & \text { MAR index } \\ 1 & \text { S. } \text { enteritidis } & \text { S, CE, E, NA, P, SXT, T, CN, CF, K, N, DO, CP, AM, AK, G } & 1 \\ 2 & \text { S. enteritidis } & \text { S, CE, E, NA, P, SXT, T, CN, CF } & 0.563 \\ 3 & \text { S. enteritidis } & \text { S, CE, E, NA, P, SXT, T, CN } & 0.500 \\ 4 & \text { S. enteritidis } & \text { S, CE, E, NA, P } & 0.312 \\ 5 & \text { S. enteritidis } & \text { S, CE, E } & 0.187 \\ 6 & \text { S. typhimurium } & \text { S, CE, E, NA, P, SXT, T, CN, CF, K, N, DO, CP, AM, AK } & 0.938 \\ 7 & \text { S. typhimurium } & \text { S, CE, E, NA, P, SXT } & 0.375 \\ 8 & \text { S. infantis } & \text { S, CE, E, NA, P, SXT, T, CN, CF, K, N, DO, CP } & 0.812 \\ 9 & \text { S. infantis } & \text { S, CE, E, NA } & 0.250 \\ 10 & \text { S. kentucky } & \text { S, CE, E, NA, P, SXT, T, CN, CF, K, N, DO } & 0.750 \\ 11 & \text { S. heidelberg } & \text { S, CE, E } & 0.187 \\ 12 & \text { S. rissen } & \text { S, CE, E } & 0.187 \\ 13 & \text { Untypable } & \text { S } & 0.062 \\ \text { Average } 0.471 & \end{array}$


Table 9: Antibiotic resistance profile of diarrhea Salmonella isolates.

\begin{tabular}{llll} 
No. & Salmonella strains & Antimicrobial resistance profile & MAR index \\
1 & S. typhimurium & S, E, NA, P, CE, CF, N, SXT, CN, K, T, AK, DO, AM, G, CP & 1 \\
\hline 2 & S. typhimurium & S, E, NA & 0.187 \\
3 & S. enteritidis & S, E, NA, P, CE, CF, N, SXT, CN, K, T, AK, DO & 0.812 \\
4 & S. infantis & S, E, NA, P, CE, CF, N, SXT, CN & 0.563 \\
\hline Average 0.640 &
\end{tabular}

DisTRIBUTION OF SOME VIRULENCE AND QACED1 GENES AMONG SALMONELLA ISOLATES

A representative detection of some Salmonella virulence and qacED1 disinfectant genes were exposed in Table 10 revealing that all 12 Salmonella serotypes harbored invA gene, while bilA, spvC, stn, and qacED1 genes were detected in $75,16.67,66.67$, and $50 \%$ of the serotypes, respectively with the acquisition of $S$. typhimurium which contained all examined genes (Figures 1,2).

Table 10: Incidence of detected virulence genes from obtained Salmonella serotypes.

$\begin{array}{llllll}\text { Virulence genes/ } & \text { invA } & \text { hilA } & \text { spvC } & \text { Stn } & \text { qacED1 } \\ \text { Salmonella sertypes } & & & & & \\ \text { S. } \text { kentucky } & + & + & - & + & - \\ \text { S. typhimurium } & + & + & + & + & + \\ \text { S. } \text { enteritidis } & + & + & - & + & + \\ \text { S. } \text { molade } & + & + & - & - & - \\ \text { S. } \text { wingrove } & + & - & - & + & - \\ \text { S. } \text { larochelle } & + & + & - & - & - \\ \text { S. } \text { inganda } & + & + & - & - & + \\ \text { S. } \text { infantis } & + & + & - & + & - \\ \text { S. } \text { tsevie } & + & + & - & + & + \\ \text { S. } \text { tamale } & + & - & - & + & - \\ \text { S. heidelberg } & + & + & - & + & + \\ \text { S. rissen } & + & - & + & - & + \\ \text { Total isolates (No.=12) } 100 \% & 75 \% & 16.67 \% & 66.67 \% 50 \%\end{array}$

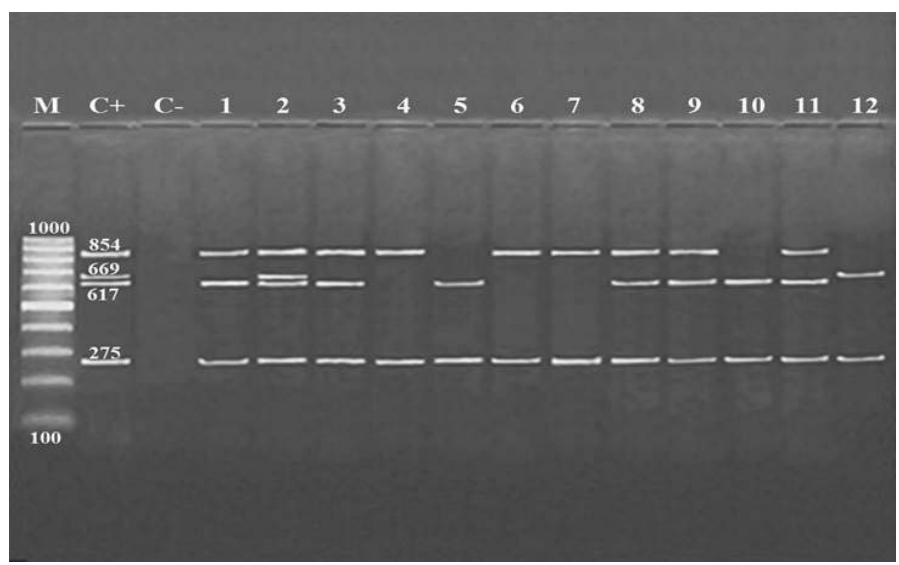

Figure 1: Agarose gel electrophoresis of multiplex PCR of invA (275 bp), stn (617 bp), spvC (669 bp) and bilA (854 bp) as virulence genes for characterization of Salmonella species.

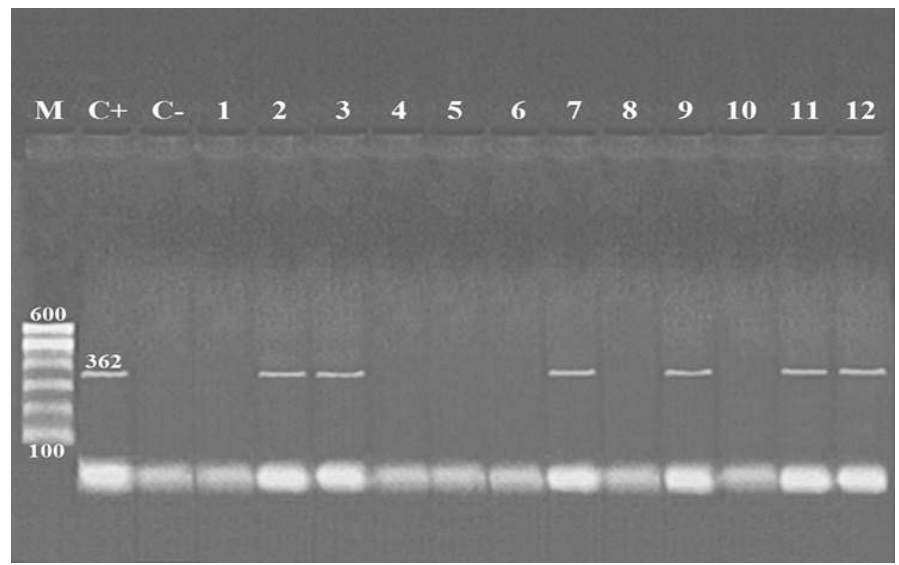

Figure 2: Agarose gel electrophoresis of PCR of qacED1 (362 bp) gene for characterization of Salmonella species.

\section{Phylogenetic analysis}

Multiple sequence alignment and phylogenesis revealed a high degree of similarities between the local isolates $(\mathrm{CH} 1$, $\mathrm{CH} 2, \mathrm{CH} 3, \mathrm{H} 1, \mathrm{H} 2$, and $\mathrm{H} 3$ ) obtained from chickenready meals and human samples, and those retrieved from the GeneBank (Figure 3).

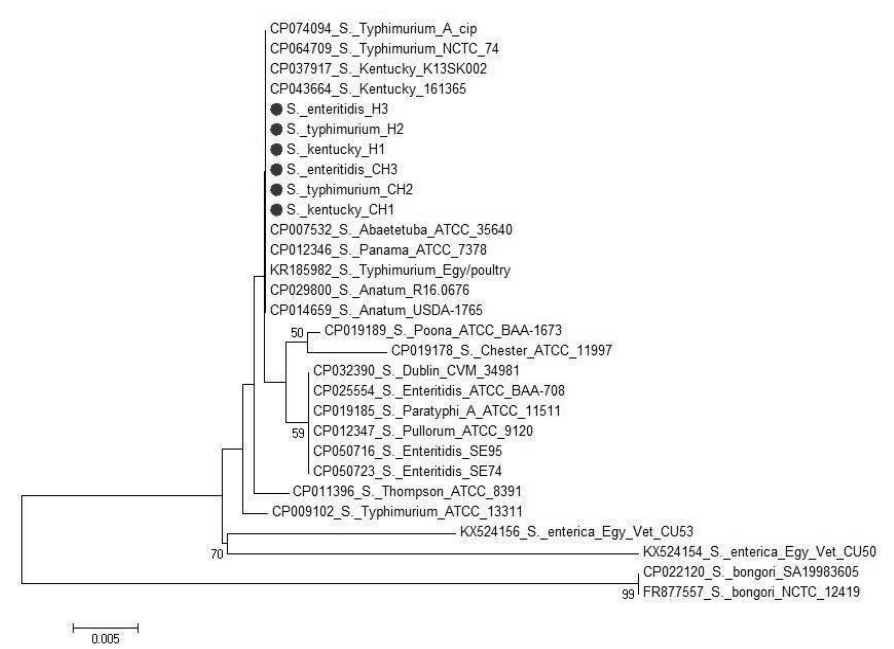

Figure 3: Phylogenetic tree of Salmonella isolated from chicken-ready meals and humans based on inv $A$ gene.

Poultry meat is one of the frequent vehicles of salmonellosis as a zoonotic infection especially ready to eat chicken meals which is in high demand as a result of their high biological value, reasonable price, and easy served. Through our study, Salmonella species were detected in $10 \%$ of the examined chicken meals with the acquisition of shish-tawook (14\%) 
which may be attributed to the fact that the temperature of grilling is not sufficient to kill micro-organisms, besides it receives more handling during preparation. This result was consistent with Abd El-Tawab et al. (2015) and Mustafa et al. (2021) who found that $10.9 \%$ and $10 \%$ of the examined chicken meat were positive for Salmonella, respectively. Other studies registered higher incidences of the organism as those conducted by Hassanin et al. (2014) (22.2\%) and Saad et al. (2015) (15\%). In contrarily, Medeiros et al. (2011); Akbar and Anal (2015) recorded lower incidences of $2.7 \%$ and $0.55 \%$, respectively. Contamination of ready meals with Salmonella might be attributed to low slaughter hygiene and cross-contamination of the products at different stages of chicken dressing and preparation in the retail shops. The variation in Salmonella incidence may be due to the differences in manufacturing practices, handling from producers to consumers and the effectiveness of hygienic measures applied during production.

Nine Salmonella serovars were isolated in this study from chicken meals which are all pathogenic to humans. $S$. typhimurium and $S$. kentucky were the predominant serotypes (20\%). In another study conducted by Elkenany et al. (2019), S. enteritidis was the most common identified serotype followed by S. typhimurium, and S. kentucky. Also, Siddique et al. (2021) discovered that $S$. typhimurium and $S$. enteritidis were the predominating types.

Controversially, there is a marked increase in antimicrobial resistance levels in developing countries as access to antimicrobials is easy and somewhat can be bought without prescription which leads to indiscriminate and widespread uses of antimicrobials both in the veterinary and public health practices (Henton et al., 2011; OIE, 2011). By evaluating antibiotic resistance in ready meals isolates, complete phenotypic resistance against erythromycin and streptomycin antibiotics with a higher resistance to cephradine (93.3\%) and sulphamethoxazol (80\%) were deemonsstrated which are commonly used in veterinary and human medicine, so considered alarming. Approximate susceptibility to ampicillin, doxycycline, and ciprofloxacin has been detected. As a result, more attention is needed towards foodborne pathogens control. In concordance with our results, Akbar and Anal (2015) found that all isolates from chicken sources were resistant to streptomycin. Siddique et al. (2021) recorded complete resistance to erythromycin and streptomycin. Contrarily, Moura et al. (2018); Perin et al. (2020) detected a high level of resistance to amoxicillin and ceftriaxone.

In the meantime, a high MAR index ranged from 0.125-1 has been observed in different food Salmonella serovars in our study, similarly, Siddique et al. (2021) detected a high index of $0.62-0.91$.
Regarding human cases, unexpectedly, our results showed a higher percentage of the microbe in food handlers hand swabs (13\%) than in patients diarrhea (8\%). So, this highlights the role played by food handlers in the transmission of food-borne diseases that represent a global health burden and they must always be under oversight. Variable incidences in poultry shops workers and food handlers were reported by Salem et al. (2017) and Yesigat et al. (2020) as $20 \%$ and $2.5 \%$, respectively. On the other hand, Rabie et al. 2012 recorded a closely related incidence of salmonellosis in patients diarrhea (10\%), while higher incidences were declared by Salem et al. (2017) (13.79\%) and Ngogo et al. (2020) (16.5\%).

Detection of Salmonella in food handlers is usually associated with untrimmed fingernails under which the bacteria locate, thus they might play a great role in the food-borne spread of salmonellosis, the result that confirmed with Phi coefficient statistic. Serotyping declared that numerous serovars could be recovered from the examined hand swabs including, S. typhimurium, S. infantis, S. kentucky, S. rissen, S. heidelberg with the acquisition of $S$. enteritidis. While one isolate couldn't be serotyped. From diarrheal samples, $S$. Enteritidis, $S$. infantis with the obsession of $S$. typhimurium have been recorded. Therefore, S. enteritidis was the dominant serotype (35\%) in the overall human samples followed by $S$. typhimurium (24\%) and $S$. Infantis (18\%). Proportionate with our result, Qi et al. (2019) and Chirambo (2020) revealed that $S$. enteritidis and $S$. typhimurium are the most common serotypes causing gastroenteritis reflecting the importance of the results obtained in this study.

A total of 150 individuals participated in this study represented by 100 food handlers and 50 hospitalized patients, at ages ranging from 5-60 years old. The majority of the participants were between 20 and 35 years. Insignificant sex risk factor was found in the current study despite the difference in the infection rate between males and females as 14.67, 8 and 4.76 and $10.34 \%$ in food handlers and patients, respectively. Compatible results were achieved by Mengist et al. (2018) and Ngogo et al. (2020). This can be explained by the fact that the incidence of infection increases with increased contact with food either among food handlers or patients. Consistent with our findings, several studies have shown that the incidence of diarrheal illness, in general, is higher in women than men.

Concerning age, a high level of Salmonella infection was reported at ages ranging from $20<35$ years among food handlers, which may be due to that this is the right age for working and so more contact with infection sources. Also, may be as a consequence of their substandard personal hygiene and lack of washing hands especially after using 
the toilet. The same result was obtained by Mengist et al. (2018) with the highest infection level at ages of 2130 years. Belonging hospitalized patients, the age group of $35<50$ showed the highest salmonellosis level which may be attributed to the feeding of undercooked foods or food contaminated after cooking during preparation or serving. Similar data was recorded by Chirambo (2020). Contrarily, patients at ages of 11-20 years exhibited the highest degree of infection followed by 21-30 age group by Teshome et al. (2019).

In the current study, human Salmonella isolates showed completeorhigher resistance to erythromycin,streptomycin, nalidixic acid, cephradine, cefotaxim, sulphamethoxazol, and penicillin $\mathrm{G}$ which may be affiliated to the unrestricted use of these antibiotics in the community. Our results were compatible with those obtained by Maripandi and AlSalamah (2010) and Singh et al. (2012). While somewhat differ from results recorded by Mengist et al. (2018) and Yesigat et al. (2020) who detected complete resistance to ampicillin. The increased resistance pattern showed by Salmonella population remains a serious public health problem and could be responsible for treatment failures in some clinical cases.

Quinolones are broad-spectrum antibiotics used in the treatment of several infections including salmonellosis particularly in the elderly and immunosuppressed patients which represented in our study by complete resistance of the human isolates to nalidixic acid while were sensitive to ciprofloxacin. High resistance to cefotaxim, the drug of choice when quinolones are contraindicated (Egorova et al., 2008), also was reported by this study giving warning about the use of antibiotics.

Salmonella virulence is influenced by antimicrobial and disinfectant resistance, as well as the presence of virulence genes. So, 12 Salmonella serotypes were analyzed for the presence of four virulence genes; invA, bilA, spvC, and $s t n$ in addition to qacED1. In all Salmonella isolates, inv $A$ gene was detected explaining their ability to invade and so causing gastroenteritis (Lan et al., 2018). Several studies reported the detection of this gene in all Salmonella species as their inner membranes contain protein coded for by invA (Amini et al., 2010; Ramatla et al., 2020).

Nine Salmonella isolates in the present study harbored bilA gene (75\%) which activates the expression of inv $A$ gene, this result was in corroboration with Borges et al. (2013) who found that all Salmonella isolates were positive for inv $A$ and bilA. $16.67 \%$ of the obtained isolates contained spvC gene, contrasting results were obtained by Soto et al. (2006) who detected it in all Salmonella isolates unlike Chaudhary et al. (2015) who could not detect that gene.
The chromosomally encoded virulent $\operatorname{stn}$ gene is widely distributed among Salmonella serovars, this data agrees with the current work since it could be detected in a percentage of 66 of the obtained isolates. Similar results were reported by Murugkar et al. (2003) and Ezzat et al. (2014).

The qacED1 gene was detected by $50 \%$ of the isolates, $\mathrm{Abd}$ El-Tawab etal.(2016) distinguished qacED1 gene in 57.14\% of Salmonella. While Nabil and Yonis (2019); Iraqi et al. (2020) detected that gene in all Salmonella representative isolates and also found a significant association between the presence of qacED1 and antimicrobial resistance.

A high homology between $\mathrm{CH} 1, \mathrm{CH} 2, \mathrm{CH} 3, \mathrm{H} 1, \mathrm{H} 2$, and $\mathrm{H} 3$ which were obtained from chicken meals, food handlers, and patients was accentuated based on inv $A$ gene sequencing, which is a very important tool for periodical evaluation of mutagenicity compared with the published sequences on GenBank. This explains the role that chicken-ready meals and food handlers play in transmitting salmonellosis to patients.

\section{CONCLUSIONS AND RECOMMENDATIONS}

This work presented a comprehensive study of salmonella presence in chicken ready meals as a major concern for human salmonellosis. The close evolutionary relationship between isolates in our study highlights the potential role of food handlers in transmitting different Salmonella serotypes to ready meals during preparation as well as to customers. Furthermore, resistance of most recovered Salmonella isolates to multiple antibiotics is of great priority. Such data impose screening of food handlers, training of hand hygiene practices, and regular monitoring of food handling practices to avoid diseases that can be acquired through improper food handling, like salmonellosis. In addition, bio-control measures must be applied to control salmonella infection within chicken farms and antibiotic resistance must be managed through enforcement of management strategies.

\section{ACKNOWLEDGMENTS}

We would like to thank the staff members of Abo-Noub Hospital for helping in the collection of patients samples as well as, workers and food handlers.

\section{NOVELTY STATEMENT}

This research sheds light on the role that food handlers play in the spread of multi-drug resistant Salmonella species through chicken-ready meals, as few studies have 
addressed the issue from a zoonotic point of view.

\section{AUTHOR'S CONTRIBUTION}

All authors contributed equally and approved the final manuscript.

\section{ETHICAL APPROVAL}

This study was approved by the South Valley University ethical committee, Qena, Egypt (No. 21/15.9.2019). Also, Oral consent was obtained from each participant.

\section{CONFLICT OF INTEREST}

The authors have declared no conflict of interest.

\section{REFERENCES}

Abd El-Tawab AA, Ammar AM, Nasef SA, El- Hofya FI, Nabil NM (2015). Studies on different Salmonella serotypes isolated from poultry in different governorates in Egypt. Benha Vet. Med. J., 28(2): 169-175. https://doi. org/10.21608/bvmj.2015.32498

Abdel-Tawab AA, Nasef SA, Ibrahim OA (2016). Bacteriological and molecular studies on bacteria causing omphalitis in chicks with regard to disinfectant resistance. Glob. Vet., 17(6): 539-545.

Akbar A, Anal AK (2015). Isolation of Salmonella from ready to eat poultry meat and evaluation of its survival at low temperature, microwaving and simulated gastric fluids. J. Food Sci. Technol., 52(5): 3051-3057. https://doi. org/10.1007/s13197-014-1354-2

Almas M, Tahir U, Zameer M, Shafiq MI, Farrukh SY, Zahra N, Mazhar M, Gill SA, Hadi F, Muhammad T, Ali Q, Malik A (2021). Detection of INVA gene and cytotoxin of Salmonella entertidis in food samples using molecular methods. J. Pharm. Res. Int., 33(12): 20-28. https://doi.org/10.9734/ jpri/2021/v33i1231249

Altschul SF, Gish W, Miller W, Myers EW, Lipmanl DJ (1990). Basic local alignment search tool. J. Mol. Biol., 215: 403410. https://doi.org/10.1016/S0022-2836(05)80360-2

Amini K, Salehi TZ, Nikbakht G, Ranjbar R, Amini J, Ashrafganjooei SB (2010). Molecular detection of invA and spv virulence genes in Salmonella enteritidis isolated from human and animals in Iran. Afr. J. Microbiol. Res., 4(21): 2202-2210.

Andino A, Hanning I (2015). Salmonella enterica: Survival, colonization, and virulence differences among serovars. Sci. World J., 2015: 520179. https://doi. org $/ 10.1155 / 2015 / 520179$

Bakheet AA, Ali NM, Al-Habaty SH, Nasef SA (2017). Detection of Disinfectant resistant aerobic bacteria in unhatched chicken eggs. Benha Vet. Med.J., 32(2): 248-259.

Borges KA, Furian TQ, Borsoi A, Moraes HL, Salle CT, Nascimento VP (2013). Detection of virulence-associated genes in Salmonella enteritidis isolates from chicken in South of Brazil. Pesqui. Vet. Bras., 33(12): 1416-1422. https://doi. org/10.1590/S0100-736X2013001200004

Card R, Vaughan K, Bagnall M, Spiropoulos J, Cooley W, Strickland $T$ (2016). Virulence characterization of Salmonella enterica isolates of differing antimicrobial resistance recovered from UK livestock and imported meat samples. Front. Microbiol., 7: 640. https://doi.org/10.3389/ fmicb.2016.00640

Castro N, Pineda E, Ochoa M (2002). Detection of hilA gene sequences in serovars of Salmonella enterica subspecies enterica. Mem. Inst. Oswaldo Cruz., 97: 1153-1156. https:// doi.org/10.1590/S0074-02762002000800016

Chai S, Cole D, Nisler A, Mahon B (2017). Poultry: The most common food in outbreaks with known pathogens, United States, 1998-2012. Epidemiol. Infect., 145: 316-325. https://doi.org/10.3382/ps/pew345

Chaudhary JH, Nayak JB, Brahmbhatt MN, Makwana PP (2015). Virulence genes detection of Salmonella serovars isolated from pork and slaughter house environment in Ahmedabad, Gujarat. Vet. World, 8(1): 121-124. https:// doi.org/10.14202/vetworld.2015.121-124

Chirambo AC (2020). Performance of molecular methods for the detection of Salmonella in human stool specimens, Version 2. Wellcome Open Res., 5: 237. https://doi.org/10.12688/ wellcomeopenres.16305.1

Chuanchuen R, Khemtong S, Padungtod P (2007). Occurrence of qace/qacED genes and their correlation with Class 1 integrons in Salmonella enterica isolates from poultry and swine. Southeast Asian J. Trop. Med. Publ. Health, 38: 855862.

CLSI (2017). Performance Standards for Antimicrobial Susceptibility Testing (27th ed.), Clinical and Laboratory Standards Institute, Wayne, USA (2017).

das Neves GB, Pick E, Giuriatti J, Araujo DN, Stefani LM (2020). Annals of medicine and medical research. Ann. Med. Med. Res., 3: 1-5.

Ed-dra A, Filali FR, Karraouan B, El Allaoui A, Aboulkacem A, Bouchrif B (2017). Prevalence, molecular and antimicrobial resistance of Salmonella isolated from sausages in Meknes. Morocco Microb. Pathog. 105: 340-345. https://doi. org/10.1016/j.micpath.2017.02.042

Egorova, SM, Timinouni M, Demartin, SA, Granier JM, Whichard V, Sangal L, Fabre A, Delaune M, Pardos Y, Millemann E, Espie M, Achtman PA, Grimont FX (2008). Ceftriaxone-resistant Salmonella enterica serotype Newport, France. Emerg. Infect. Dis., 14: 954-957. https://doi. org/10.3201/eid1406.071168

Elkenany R,Elsayed MM, Zakaria AI, El-Sayed SA, Abdo Rizk M (2019). Antimicrobial resistance profiles and virulence genotyping of Salmonella enterica serovars recovered from broiler chickens and chicken carcasses in Egypt. BMC Vet. Res., 15(1): 124. https://doi.org/10.1186/s12917-0191867-z

El-Sharkawy H, Tahoun A, El-Gohary AEGA, El-Abasy M, El-Khayat F, Gillespie T (2017). Epidemiological, molecular characterization and antibiotic resistance of Salmonella enterica serovars isolated from chicken farms in Egypt. Gut Pathog., 9: 8. https://doi.org/10.1186/s13099-017-0157-1

Ezzat ME, Shabana II, Esawy AM, Elsotohy ME (2014). Detection of virulence genes in Salmonella serovars isolated from broilers. Anim. Vet. Sci., 2(6): 189-193. https://doi. org/10.11648/j.avs.20140206.16

Favier GI, Estrada CSL, Otero VL, Escudero ME (2013). Prevalence, antimicrobial susceptibility, and molecular characterization by PCR and pulsed field gel electrophoresis (PFGE) of Salmonella spp. isolated from foods of animal origin in San Luis, Argentina. Food Contr., 29: 49-54. https://doi.org/10.1016/j.foodcont.2012.05.056 
Fekry E, Ammar AM, Hussien A (2018). Molecular detection of InvA, OmpA and Stn genes in Salmonella serovars from broilers in Egypt. Alex. J. Vet. Sci., 56: 69-74. https://doi. org/10.5455/ajvs.288089

Gracias KS, McKillip JL (2004). A review of conventional detection and enumeration methods for pathogenic bacteria in food. Can. J. Microbiol., 50(11): 883-890. https://doi. org/10.1139/w04-080

Hassanin FS, Reham A, Amin Shawky NA, Gomaa WM (2014). Incidence of Escherichia coli and Salmonella in ready to eat foods. Benha Vet. Med. J., 27(1): 84-91.

Haynes RL, Smith TW (2003). Hatchery management guide for game birds and small poultry flock owners. A 7-page online publication of Mississippi State University. Accessed 20 $0^{\text {th }}$ June.

Henton M, Eagar H, Swan G, Vuuren M (2011). Antibiotic management and resistance in livestock production. South Afr. Med.J., 101: 8.

Iraqi M, Nasef SA, El-Enbaawy M (2020). Early broiler chicks' mortalities: Phenotypic and genotypic characters of antimicrobial and disinfectant resistance of gram-negative bacteria involved in such mortalities. Int. J. Vet. Sci., 10(2): 129-134. https://doi.org/10.47278/journal.ijvs/2020.033

ISO (2002). International organization for standardization. No. 6579. Microbiology of food and animal feeding stuffs Horizontal methods for detection of Salmonella species.

Kauffman F (1974). Serological diagnosis of Salmonella species. Kauffman White Scheme, Copenhagen, Denmark.

Lan TT, Gaucher ML, Nhan NT, Letellier A, Quessy S (2018). Distribution of virulence genes among Salmonella serotypes isolated from pigs in Southern Vietnam. J. Food Prot., 81(9): 1459-1466. https://doi.org/10.4315/0362-028X.JFP-17408

Maripandi A, Al-Salamah AA (2010). Multiple-antibiotic resistance and plasmid profiles of Salmonella enteritidis isolated from retail chicken meats. Am. J. Food Technol., 5(4): 260-268. https://doi.org/10.3923/ajft.2010.260.268

Medeiros MAN, Oliveira DCNd, Rodrigues DdP, Freitas DRCd (2011). Prevalence and antimicrobial resistance of Salmonella in chicken carcasses at retail in 15 Brazilian cities. Rev. Panam. Salud Pública, 30(6): 555-560. https:// doi.org/10.1590/S1020-49892011001200010

Mengist A, Mengistu G, Reta A (2018). Prevalence and antimicrobial susceptibility pattern of Salmonella and Shigella among food handlers in catering establishments in Debre Markos University, Northwest Ethiopia. Int. J. Infect. Dis., 75: 74-79. https://doi.org/10.1016/j.ijid.2018.08.008

Moura Q,Fernandes MR, Silva KC,Monte DF, Esposito F, Dropa M (2018). Virulent nontyphoidal Salmonella producing CTX-M and CMY-2 $\beta$-lactamases from livestock, food and human infection. Braz. Virulence, 9: 281-286. https://doi.or $\mathrm{g} / 10.1080 / 21505594.2017 .1279779$

Munck N, Smith J, Bates J, Glass K, Hald T, Kirk MD (2020). Source attribution of Salmonella in Macadamia nuts to animal and environmental reservoirs in Queensland, Australia. Foodborne Pathog. Dis., 17: 357-364. https://doi. org/10.1089/fpd.2019.2706

Murugkar HV, Rahman H, Dutta PK (2003). Distribution of virulence genes in Salmonella serovars isolated from man and animals. Indian J. Med. Res., 117: 66-70.

Mustafa GR, Zhao K, He X, Chen S, Liu S, Mustafa A, He L, Yang Y, Yu X, Penttinen P, Ao X, Liu A, Shabbir MZ, $\mathrm{Xu}$ X, Zou L (2021). Heavy metal resistance in Salmonella typhimurium and its association with disinfectant and antibiotic resistance. Front. Microbiol., 12: 702725. https:// doi.org/10.3389/fmicb.2021.702725

Nabil NM, Yonis AE (2019). Isolation of Salmonella characterized by biofilm formation and disinfectant resistance from broiler chickens. Alex. J. Vet. Sci., 62: 26-36. https://doi. org/10.5455/ajvs.57274

National Committee for Clinical Laboratory Standards (CLSI) (2017). Performance standards for antimicrobial susceptibility testing (27 th ed.). Clinical and Laboratory Standards Institute. Wayne, USA.

Nayak R, Stewart T, Wang RF, Lin J, Cerniglia CE, Kenney PB (2004). Genetic diversity and virulence gene determinants of antibiotic-resistant Salmonella isolated from preharvest turkey production sources. Int. J. Food Microbiol., 91: 5162. https://doi.org/10.1016/S0168-1605(03)00330-1

Ngogo FA, Joachim A, Abade AM, Rumisha SF, Mizinduko MM, Majig MV (2020). Factors associated with Salmonella infection in patients with gastrointestinal complaints seeking health care at Regional Hospital in Southern Highland of Tanzania. BMC Infect. Dis., 20: 135. https:// doi.org/10.1186/s12879-020-4849-7

OIE (2011). The OIE regional commission for Africa. In proceedings of the $14^{\text {th }}$ Conference of the OIE, Tehran, Iran, November 2011.

Perin AP, Martins BTF, Barreiros MAB, Yamatogi RS, Nero LA, dos Santos Bersot L (2020). Occurrence, quantification, pulse types, and antimicrobial susceptibility of Salmonella sp. isolated from chicken meat in the state of Paraná, Brazil. Braz. J. Microbiol., 51: 335-345. https://doi.org/10.1007/ s42770-019-00188-X

Qi X, Li P, Xu X, Yuan Y, Bu S, Lin D (2019). Epidemiological and molecular investigations on Salmonella responsible for gastrointestinal infections in the southwest of Shanghai from 1998 to 2017. Front. Microbiol., 10: 2025. https://doi. $\mathrm{org} / 10.3389 /$ fmicb. 2019.02025

Rabie NS, Khalifa NO, Radwan ME, Afify JS (2012). Epidemiological and Molecular Studies of Salmonella Isolates from Chicken, Chicken Meat and Human in Toukh, Egypt. Glob. Vet., 8(2): 128-132.

Ramatla TA, Mphuthi N, Ramaili T, Taioe MO, Thekisoe OMM, Syakalima M (2020). Molecular detection of virulence genes in Salmonella spp. isolated from chicken faeces in Mafikeng, South Africa.J. South Afr. Vet. Assoc., 91: 1994. https://doi. org/10.4102/jsava.v91i0.1994

Rodrigues GL, Panzenhagen P, Ferrari RG, dos Santos A, Paschoalin VMF, Conte-Junior CA (2020). Frequency of antimicrobial resistance genes in Salmonella from brazil by in silico whole-genome sequencing analysis: An overview of the last four decades. Front. Microbiol., 11: 1864. https:// doi.org/10.3389/fmicb.2020.01864

Saad SM, Edris AM Hassan MA, Edris SNM (2015). Antibiotic sensitivity of Salmonella species isolated from chicken meat products. Benha Vet. Med. J., 28(2): 141-146. https://doi. org/10.21608/bvmj.2015.32491

Salem L, Awadallah M, Nashw OK, Salem M (2017). Zoonotic importance of Salmonellosis in chickens and humans at Qualyobia Province. Egypt. J. Vet. Sci., 47(2): 151-164. https://doi.org/10.21608/ejvs.2016.3585

Siddique A, Azim S, Ali A, Andleeb S, Ahsan A, Imran M, Rahman A (2021). Antimicrobial resistance profiling of biofilm forming non typhoidal Salmonella enterica isolates from poultry and its associated food products from 
Pakistan. Antibiotics, 10: 785. https://doi.org/10.3390/ antibiotics 10070785

Singh S, Agarwal RK, Tiwari SC, Singh H (2012). Antibiotic resistance pattern among the Salmonella isolated from human, animal and meat in India. Trop. Anim. Health Prod., 44: 665-674. https://doi.org/10.1007/s11250-011-9953-7

Sodagari, HR, Wang P, Robertson I, Habib I, Sahibzada S (2020). Non-typhoidal Salmonella at the human food of animal origin interface in Australia. Animals, 10: 1192. https://doi. org/10.3390/ani10071192

Soto SM, Rodriguez I, Rodicio MR, Vila J, Mendoza MC (2006). Detection of virulence determinants in clinical strains of Salmonella enterica serovar Enteritidis and mapping on macrorestriction profiles. J. Med. Microbiol., 55: 365-373. https://doi.org/10.1099/jmm.0.46257-0

Swamy S, Barnhart H, Lee M, Dreesen D (1996). Virulence determinants invA and spvC in Salmonellae isolated from poultry products, wastewater, and human sources. Appl. Environ. Microbiol., 62: 3768-3771. https://doi. org/10.1128/aem.62.10.3768-3771.1996

Takaya A, Yamamoto T, Tokoyoda K (2020). Humoral immunity vs. Salmonella. Front. Immunol., 10: 3155. https://doi. org/10.3389/fimmu.2019.03155

Tamura K, Stecher G, Peterson D, Filipski A, Kumar S (2013). MEGA6: Molecular evolutionary genetics analysis version 6.0. Mol. Biol. Evol., 30: 2725-2729. https://doi. org $/ 10.1093 / \mathrm{molbev} / \mathrm{mst197}$

Techaruvichit P, Takahashi H, Kuda T, Miya S, Keeratipibul
S, Kimura B (2016). Adaptation of Campylobacter jejuni to biocides used in the food industry affects biofilm structure, adhesion strength, and cross-resistance to clinical antimicrobial compounds. Biofouling, 32(7): 827-839. https://doi.org/10.1080/08927014.2016.1198476

Teshome B, Teklemariam Z, Ayana DA, Marami D, Asaminew N (2019). Salmonella and Shigella among patients with diarrhea at public health facilities in Adama, Ethiopia: Prevalence, antimicrobial susceptibility pattern, and associated factors. SAGE Open Med., 7: https://doi. org/10.1177/2050312119846041

WHO (2020). Food safety. Geneva: WHO.

Xu H, Lee HY, Ahn J (2010). Growth and virulence properties of biofilm-forming Salmonella enterica serovar Typhimurium under different acidic conditions. Appl. Environ. Microbiol., 76(24): 7910-7917. https://doi.org/10.1128/AEM.0150810

Yesigat T, Jemal M, Birhan W (2020). Prevalence and associated risk factors of Salmonella, Shigella, and intestinal parasites among food handlers in Motta Town, North West Ethiopia. Can. J. Infect. Dis. Med. Microbiol., Volume 2020 |Article ID 6425946. https://doi.org/10.1155/2020/6425946

Zou L, Meng J, McDermott PF, Wang F, Yang Q, Cao G, Hoffmann M, Zhao S (2014). Presence of disinfectant resistance genes in Escherichia coli isolated from retail meats in the USA. J. Antimicrob. Chemother., 69: 26442649. https://doi.org/10.1093/jac/dku197 